\title{
Simulation of nonlinear superconducting rf losses derived from characteristic topography of etched and electropolished niobium surfaces
}

\author{
Chen Xu, ${ }^{1,2}$ Charles E. Reece, ${ }^{1}$ and Michael J. Kelley ${ }^{1,2}$ \\ ${ }^{1}$ Thomas Jefferson National Accelerator Facility, Newport News, Virginia 23606, USA \\ ${ }^{2}$ Department of Applied Science, The College of William \& Mary, Williamsburg, Virginia 23185, USA \\ (Received 10 June 2014; revised manuscript received 29 August 2015; published 22 March 2016)
}

A simplified numerical model has been developed to simulate nonlinear superconducting radiofrequency (SRF) losses on $\mathrm{Nb}$ surfaces. This study focuses exclusively on excessive surface resistance $\left(R_{s}\right)$ losses due to the microscopic topographical magnetic field enhancements. When the enhanced local surface magnetic field exceeds the superconducting critical transition magnetic field $H_{c}$, small volumes of surface material may become normal conducting and increase the effective surface resistance without inducing a quench. We seek to build an improved quantitative characterization of this qualitative model. Using topographic data from typical buffered chemical polish (BCP)- and electropolish (EP)-treated fine grain niobium, we have estimated the resulting field-dependent losses and extrapolated this model to the implications for cavity performance. The model predictions correspond well to the characteristic BCP versus EP high field $Q_{0}$ performance differences for fine grain niobium. We describe the algorithm of the model, its limitations, and the effects of this nonlinear loss contribution on SRF cavity performance.

DOI: 10.1103/PhysRevAccelBeams.19.033501

\section{INTRODUCTION}

The roughness of superconducting radiofrequency (SRF) surfaces has long been recognized as influential on mechanisms which limit the performance of niobium SRF resonators [1]. Decreasing cavity unloaded quality factor $Q_{0}$ reflects an increasing of average surface resistance, $R_{s}$. Several models attempt to explain the dependency of quality factors at different accelerator fields. Agreement of these models and experiments has been mixed [2]. Surface roughness has been associated with increased losses, lower quench fields, and increased difficulty in cleaning. Various surface treatments are implemented to achieve beneficial smoothness. Typical surfaces have been statistically characterized and analyzed. Different treatments modify surface features at various lateral length scales $[3,4]$. However, it has not been well established just how the details of topographical features directly affect integrated $\mathrm{rf}$ performance. It is understood that sharp features promote magnetic field enhancement and may, under appropriate conditions, initiate quench $[5,6]$.

Knobloch et al. estimated if loss from a grain boundary edge and extended this estimate to anticipated effects in an SRF cavity [5]. Here, this method is improved by a detailed finite element method simulation. This simulation also integrates both rf field and thermal calculations on representative niobium SRF surfaces obtained by AFM

Published by the American Physical Society under the terms of the Creative Commons Attribution 3.0 License. Further distribution of this work must maintain attribution to the author $(s)$ and the published article's title, journal citation, and DOI. profilometry with micrometer resolution rather than infer a distribution function of local field enhancements from observed cavity performance constraints. In addition, we allow the size of local normal conducting volumes to be determined dynamically, rather than assuming a fixed width and depth as was done in [5]. Second, this simulation incorporates the temperature dependency of various superconducting material properties. We customized an algorithm to iteratively compute rf losses under steady state conditions. Our analysis provides for no fit parameters, just direct calculation limited by the available mesh resolution. Such an attempt to model increased rf losses due to topographic enhancements has not been previously reported. Third, we will relate the simulation results to accelerator cavity performance differences associated with either chemical etching or electropolishing finishing steps.

Typical buffered chemical polish (BCP)-treated fine grain $\mathrm{Nb}$ cavities commonly show a $Q_{0}$ that starts to decrease with dramatic slope when the accelerating gradient increases from 16 to $22 \mathrm{MV} / \mathrm{m}$. This occurs even after the cavities are treated with a postchemistry bake [7-9]. In some extreme cases, cavities exhibit this kind of nonlinear loss when the accelerating gradient is as low as $15 \mathrm{MV} / \mathrm{m}$. After electropolish (EP) treatment, this $Q_{0}$ decrease is removed [9-11]. This frequently encountered phenomenon is dramatically illustrated in Fig. 1, which presents the performance of Jefferson Lab CEBAF sevencell prototype cavity HG006 with very heavy BCP etch followed by a $30 \mu \mathrm{m} \mathrm{EP}$, with no field emission loading in any test [12]. Such a difference in performance has come to be qualitatively associated with field enhancements of the "rougher" BCP-treated surface. Such roughness from 


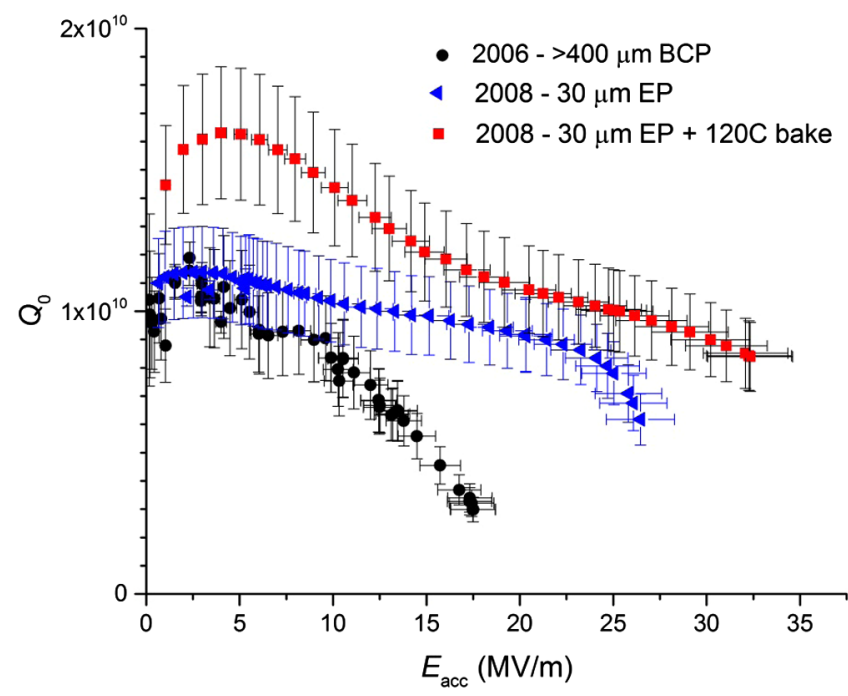

FIG. 1. Performance of $\mathrm{Nb}$ cavity HG006 after a very heavy etching BCP and a subsequent $30 \mu \mathrm{m}$ EP [12].

chemical etching can be highly variable depending on crystalline structure and defect density of the niobium surface and amount of material removal. Since the principal difference between these two surface states is microscopic topographical roughness, these results suggest that managing topography evolution plays a critical role in improving useful cavity gradient.

Compared to EP surfaces, BCP-treated surfaces have a higher root mean square (rms) height variation and a greater density of sharp features [3,4]. Those high and sharp features enhance the local magnetic field, and the enhanced magnetic field at these local features may exceed the superconducting critical field $H_{\text {crit }}$. As a result, local superconducting transition is initiated and small areas may become normal conducting. For niobium, in general a mixed state occurs and transition is quite complicated. As a weak type II superconductor, $\mathrm{Nb}$ has a Ginzburg-Landau factor around 1.3, close to type I superconductors [13].

In this paper, we focus our study on the topographical enhancements to surface rf losses. For simplification, we simply treat $\mathrm{Nb}$ as a type I superconductor. Thus, a mixed state will be ignored and $\mathrm{Nb}$ will become normal conducting whenever the local field exceeds $H_{c}$, as a type I superconductor. We choose to simplify the normal to superconducting transition by using a single value $H_{\text {crit }}$ to identify normal and superconducting boundary. In a more complex case, a section of material under the surface will be in a mixed state when $H_{\text {crit }}$ is reached. Thus the normal and superconducting boundary will become a belt rather than a line. This belt volume represents the mixed state. With added complexity one could calculate the rf loss within this belt and the normal-conducting zones. We leave that effort for future research. In this analysis, the precise value of $H_{\text {crit }}$ is open for discussion; somewhat arbitrarily we use the superheating field $H_{\text {sh }}$ in our simulations [14].
Note that this $H_{\text {sh }}$ changes dynamically according to the local temperature and also may be suppressed via the mechanism described by Kubo [15]. Uncertainty in the precise value of the effective $H_{\text {crit }}$ has little impact on the resulting analysis of the present paper, but remains an opportunity for further future refinement.

The local effective field enhancement may be quantified by the local geometric magnetic field enhancement factor (LGMFE). This index is a ratio of local enhanced magnetic field over the nominally applied rf field $[16,17]$.

The magnetic field amplitude decays exponentially in the $\mathrm{Nb}$ material. When the surface $H$ field is greater than $H_{c}$, a location inside the surface will have an $H$ field less than $H_{c}$. In this circumstance, there is an interface between normal conducting material on the surface and superconducting material in the bulk. Because the electric time constants are so short compared with the rf frequency, this interface is moving along with the rf phase in our simulation relevant to $1-2 \mathrm{GHz}$ cavities. An excess rf loss is generated by these small normal nucleation sites on the surface. Moreover, this rf loss raises the local surface temperature and consequently reduces the local $H_{c}$. The positive thermal feedback aggravates the normal conducting transition. Detailed calculation is needed to evaluate the local rf loss and attendant temperature rise. The consequential additional rf loss can be expressed as an increasing effective surface resistance $[18,19]$. In addition, a temperature map may be calculated to estimate the local $H_{c}$. Temperature rise would increase the normal zones and bring additional loss. In this analysis, electromagnetic and thermal iterations are adapted to mimic this thermal feedback condition. Stable solutions are approached with a convergence. We propose a model to calculate nonlinear rf loss from microscopic surface topographical features. An averaged surface resistance as a function of applied $H$ field is given to compare with cavity cold testing experiments.

In this analysis, electromagnetic and thermal simulations are numerically provided by the finite element method (FEM). Corresponding field-dependent rf Ohmic losses are characterized from surface topography associated with two types of popular surface treatments. The effective $R_{s}$ values are calculated, and corresponding quality factor, $Q$, versus accelerating gradient, $E$, curves are generated from this analytic model. The model may be applied to cavities with various surface treatments in order to further understand and predict the influence of surface topography on practical resonators at high surface magnetic fields.

\section{METHODOLOGY}

\section{A. Electromagnetic calculation}

To calculate the electromagnetic field distribution near a surface, Maxwell's equations must be solved with a boundary condition by an eigenmode solver [20]. We 
reorganize the Maxwell equations into a Helmholtz equation as shown:

$$
\left(\nabla^{2}+k^{2}\right) \varphi=0
$$

where $\varphi$ is the magnetic scalar potential and wave number $k=|k|=\frac{\omega}{c}$.

After separation of variables, space $\phi(r)$ and time $T(t)$ give general wave solutions. An example of $1 \mathrm{D}$ solution is expressed below:

$$
\begin{aligned}
\varphi(r) & =\sum_{n} C_{1} e^{i k_{n} \cdot r}+\sum_{n} C_{2} e^{-i k_{n} \cdot r} \\
T(t) & =\sum_{n} D_{1} e^{i \omega_{n} \cdot t}+\sum_{n} D_{2} e^{-i \omega_{n} \cdot t} .
\end{aligned}
$$

In our case, we simplify the wave equation into a static form near the surface. The simplification is appropriate when the second term in Eq. (1) is much smaller than the first term. This is applicable when the simulated area lateral size is much smaller than the rf wavelength. In our simulation, the lateral scale $l$ is $100 \mu \mathrm{m}$ while wavelength $\lambda$ at $1.5 \mathrm{GHz}$ is $20 \mathrm{~cm}$. At this simulated scale, the wave number $k$ has an order $10^{-2} \mathrm{~cm}^{-1}$. The sinusoidal field difference within the simulated length is trivial. Therefore, the dominating equation reduces into a Laplace equation, given in Eq. (3):

$$
\nabla^{2} \varphi=0
$$

FEM and conformal mapping methods are used to solve the Laplace equation in 2D. The rf $H$ field gets enhanced when it crosses the groove features and remains uniform when the $H$ field comes along the groove direction. Note that $\varphi$ in Eqs. (2) and (3) can be interchanged with any vector fields and scalar potentials, such as electric field $E$, magnetic field $H$, magnetic flux $B$, magnetic scalar potential $\varphi$ or magnetic vector potential $A$. In this study, we use magnetic scalar potential $\varphi$, because it has a set of simple boundary conditions.

We take a representative surface strip profile obtained from AFM characterization of a fine grain $\mathrm{Nb}$ surface. This surface is reasonably presumed isotropic at a scale of $100 \mu \mathrm{m}$ since the typical fine grain $\mathrm{Nb}$ has grain size $20-50 \mu \mathrm{m}$. The boundaries of our model to describe the magnetic scalar potential, $\varphi$, near this surface are labeled with numbers in Fig. 2. Boundaries 1 and 3 are a pair of periodic boundary conditions. We assign them Dirichlet boundary conditions where two arbitrary magnetic scalar amplitudes are given. These two values determine the applied parallel $H$ field far from the surface. This parallel $H$ field has a range from 80 to $210 \mathrm{mT}$ in this simulation. Boundary conditions for boundaries 2 and 4 are the Neumann boundary conditions because they are treated as perfect electric walls. Boundary 4 is a surface

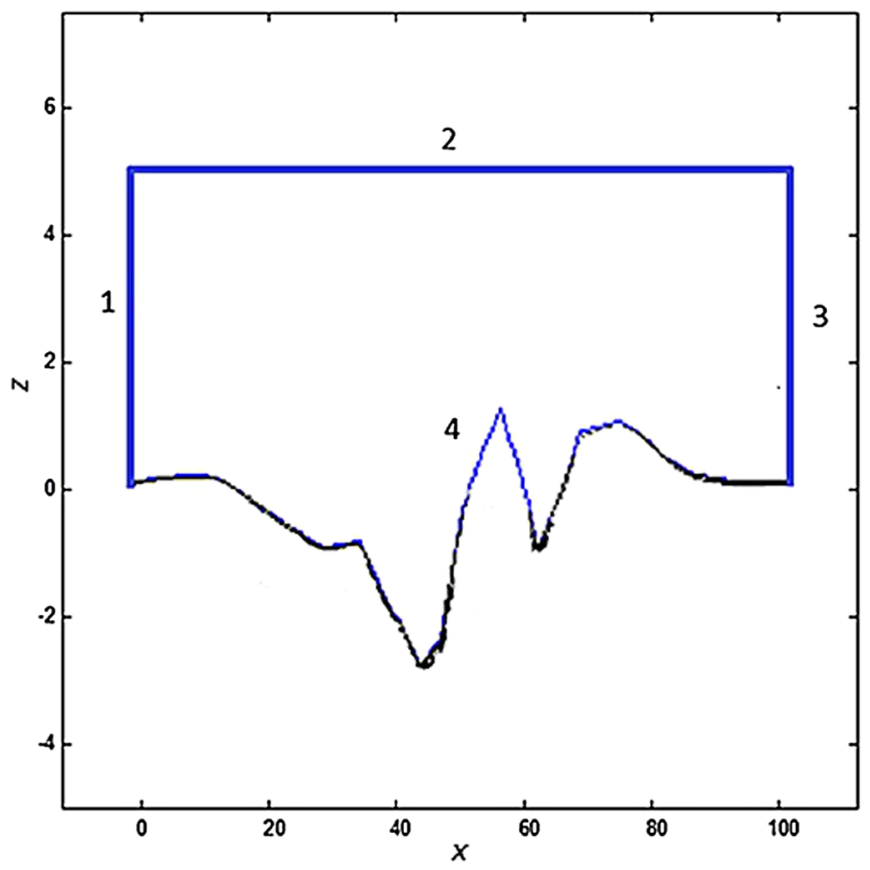

FIG. 2. Configuration used for the simulation model calculation on a cross-section fragment of a BCP-treated sample surface. The area bounded in blue represents vacuum volume. Borderlines 1 and 3 determine the exciting magnetic field. Borderline 2 and 4 are perfect electric conductors (PEC), while border 4 outlines a BCP-treated surface profile, also PEC, acquired by AFM scanning. Unit: $\mu \mathrm{m}$.

characterized by AFM from a practical BCP-treated sample. In this analysis, we take $H_{c}=190 \mathrm{mT}$ [14].

In Fig. 3, the conformal mapping calculation yields the nominally horizontal solid lines as $E$ equipotential contours, while the vertical dotted lines are magnetic equipotential contours. The magnetic field at each point on the surface $H_{\text {enhanced }}(x)$ may be calculated as a function of horizontal position $(x)$ by FEM. The solution obtained is the maximum amplitude of surface magnetic potential. The time dependent term must be added to represent the rf phase variation. The accuracy of the calculation is related to the surface characterization sampling and FEM resolution.

After FEM calculation, the magnetic field on the surface is computed by taking the derivative of the scalar potential along the surface. The LGMEF indexes along the horizontal $\mathrm{x}$ are plotted in Fig. 4. The LGMEF factors are observed varying from 0.4 to 1.9 . These amplitudes are attributed to the local surface topographic "sharpness." The LGMFE factor is greater than 1 on surface protrusions and smaller than 1 on valley areas.

When the applied magnetic field increases, the local $H$ field may begin to exceed $H_{c}$ at some surface areas where normal zones begin to nucleate. There then exists a normal and superconducting interface beneath the surface. In this study, we presume the superconducting to normal conducting transition would follow the change of the rf field 


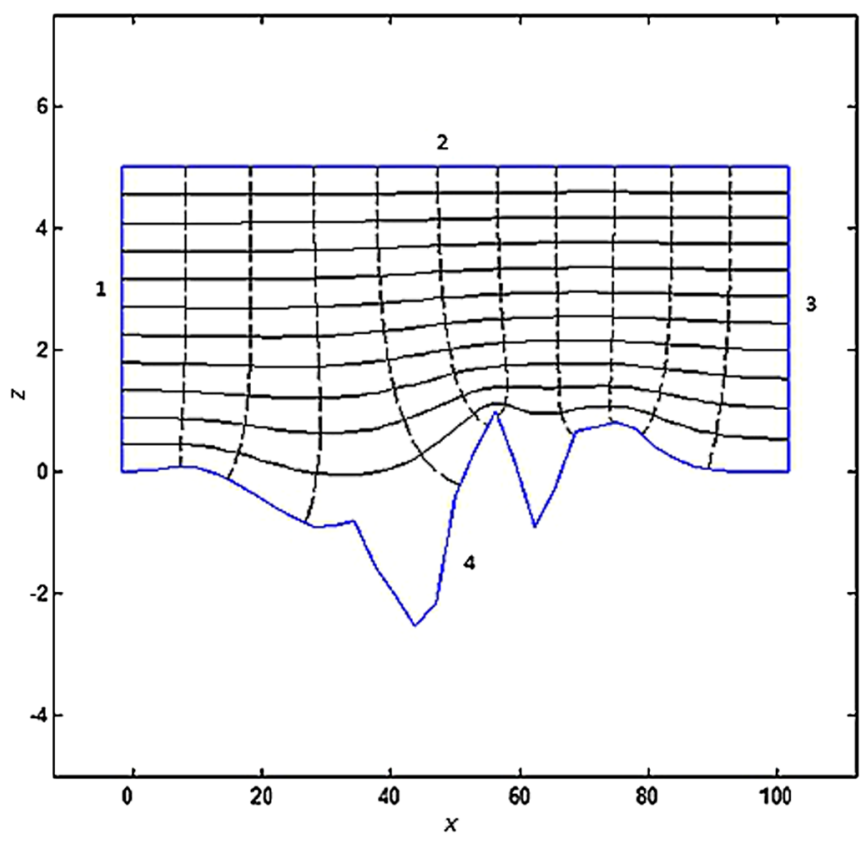

FIG. 3. Electromagnetic equipotential contours by conformal mapping calculation. The vertical dashed lines are $E$ field lines, and horizontal lines are magnetic field lines. Unit: $\mu \mathrm{m}$.

magnitude. Thus, this interface moves inward and outward with $\mathrm{rf}$ phase. The location of this interface is determined by FEM at each snapshot. Mathematically, this situation is widely known as the Stefan moving boundary problem, and it simulates surface crystallization processes and other phase transition problems [21]. An additional borderline 5 is introduced on Fig. 5. This outline 5 represents the interface, which we term the "normal conducting phase front." The tangential magnetic field value on this boundary is equal to the local $H_{c}$. Boundary 4 is subsequently ignored, because $H$ field decay within the normal zones between outlines 4 and 5 is negligible. The rest of the

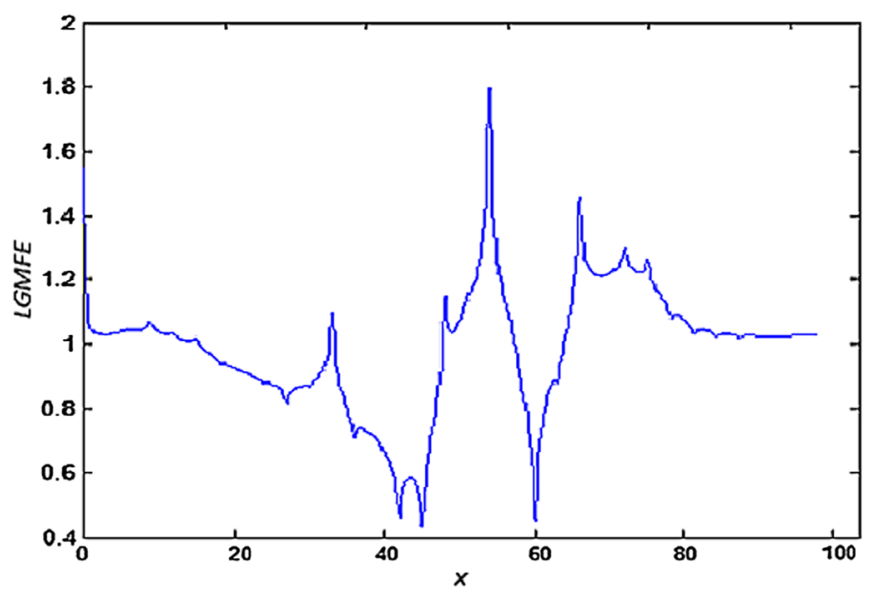

FIG. 4. The LGMFE map is calculated from the profile in Fig. 2. These indexes do not change with the applied field. Unit: $\mu \mathrm{m}$.

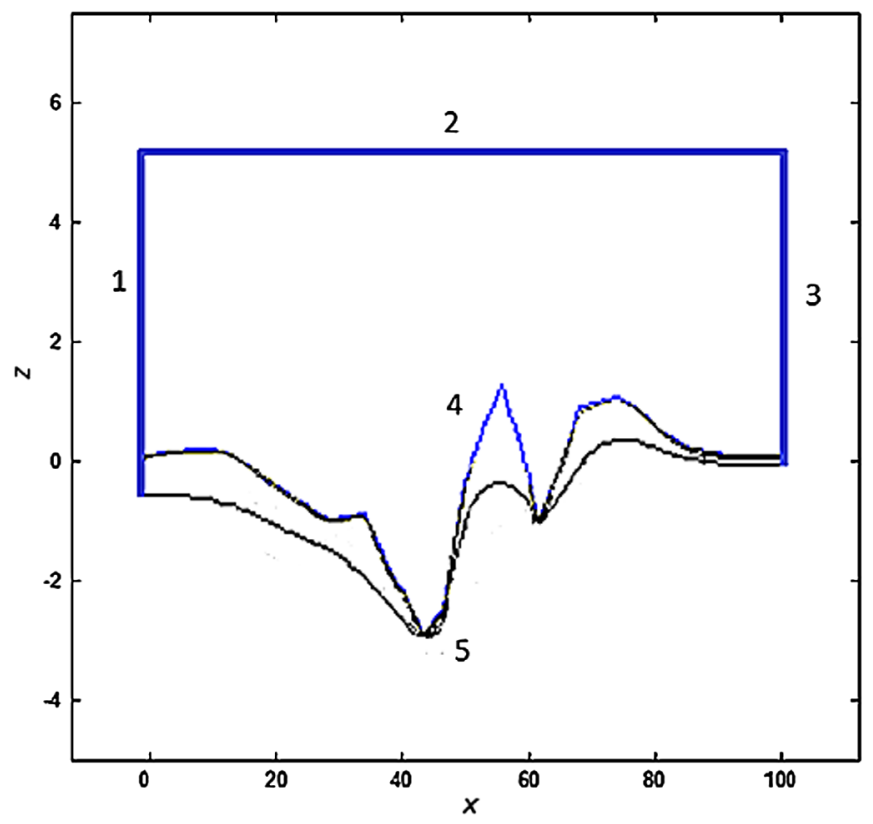

FIG. 5. Configuration used for simulation model calculation on cross section fragment of the real BCP-treated sample surface. The area bounded in blue represents vacuum volume. Borderlines 1 and 3 determine the exciting magnetic field. Borderline 2 has a PEC boundary condition. Additional border 5 defines an interface of normal and superconducting materials and also is PEC. Unit: $\mu \mathrm{m}$.

configuration in Fig. 2 remains unchanged. Conditions on boundary 5 are expressed in Eq. (4). In the particular instance illustrated in Fig. 5, the applied $H$ is greater than $H_{c}$ for clarity:

$$
\begin{gathered}
H_{\perp}=0 \\
H_{\|}=H_{\text {critical }}
\end{gathered} \quad \text { on boundary } 5 .
$$

\section{Iteration method}

Now let us discuss how to determine the location of this phase front. The basic algorithm is an iterative simulation until the known boundary conditions on the moving

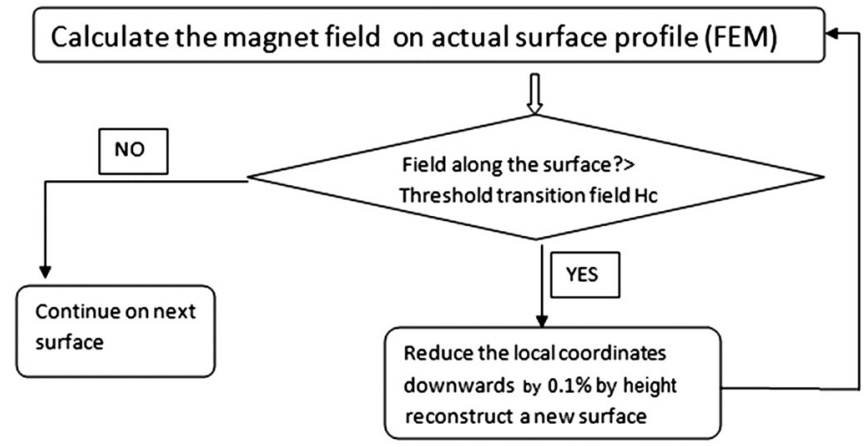

FIG. 6. Flowchart to determine the interface between the normal conducting and superconducting conducting materials. 
boundary are locally matched. The boundary conditions on this moving contour are listed in Eq. (4). Starting from the physical surface, one can calculate surface $H$ field at a given applied field. If any location on this changing boundary field has a local $H$ field greater than $H_{c}$, then the next step is to reduce this local surface height by a certain small amount. Continue calculating the field on this moving boundary until the local field on this boundary is equal to or less than $H_{c}$. Presumably, the $H$ field decay is negligible within the very shallow depth of the normal conducting zones. This is a reasonable assumption when the zone depth is a small fraction of the normal conducting skin depth. Localization of this phase front is thus calculated within certain error limitations. Accuracy can be improved at the expense of computing time. The iteration method is illustrated in the flowchart of Fig. 6.

\section{Simulation results and comparison}

Figure 7 provides the simulation results of the normal conducting phase front's deepest penetration when the amplitude of applied $H$ field ranges from 100 to $180 \mathrm{mT}$. The areas between the red and blue lines indicate the maximum normal zone volumes during each rf cycle.

At low field, there is no normal zone because the local field is weaker than $H_{c}$. For example, if the highest LGMFE index on a surface is 1.8 and $H_{c}$ is taken as $190 \mathrm{mT}$, the normal zone would be expected to nucleate when the applied $H$ field reaches $105 \mathrm{mT}$.

\section{B. Thermal simulation and its correction iteration \\ 1. Heat equation}

In this study, a thermal calculation uses the results from the electromagnetic simulation as input. This input includes the normal conducting/superconducting phase front location and $H$ field distribution. A goal for this thermal simulation is to generate a temperature map internal to the $\mathrm{Nb}$ from the rf surface to the external helium bath. The simulation estimates a temperature map in order to determine the material's phase, thermal conductivity, and dissipative losses in a self-consistent way.

After using the electromagnetic simulation results to obtain a temperature map, one can reassign the temperatures back to the material at each position to then determine the thermal conductivity. The change of thermal conductivity initiates the next round of temperature simulation. This iteration method may modify the normal-conducting phase front location results from the electromagnetic simulation, especially if the temperature of the normal conducting and superconducting interface is higher than $T_{c}$. In this thermal study, a second FEM computational code was developed to estimate the temperatures.

Thermal diffusion is governed by the partial differential equation:

$$
\frac{\partial T}{\partial t}=\operatorname{div}[\alpha(T) \cdot \nabla T]+\tilde{q},
$$

where $T$ is temperature, $\tilde{q}$ is related to the internal heat source density, and $\alpha$ is the thermal diffusivity. Note that this diffusivity is a function of temperature.

Additionally, the internal heat source density can be further expressed as

$$
\tilde{q}(t, x, y, z)=\frac{\tilde{Q}(t, x, y, z)}{\rho C_{P}} \quad \text { and } \quad \alpha=\frac{\kappa}{\rho C_{p}},
$$

where $\tilde{Q}$ is the heat generated at a given position and time, $\kappa$ is the thermal conductivity, $\rho$ is $\mathrm{Nb}$ density, $C_{p}$ is specific heat capacity, and $t$ is time. This heat is generated by rf loss on the surfaces.

For the static state solution, Eq. (5) reduces into

$$
\operatorname{div}[\alpha(T) \cdot \nabla T]=\alpha(T) \nabla^{2} T+(\nabla T)^{2} \frac{\partial \alpha}{\partial T}=-\tilde{q} .
$$

Note that the thermal conductivity is also temperature dependent.

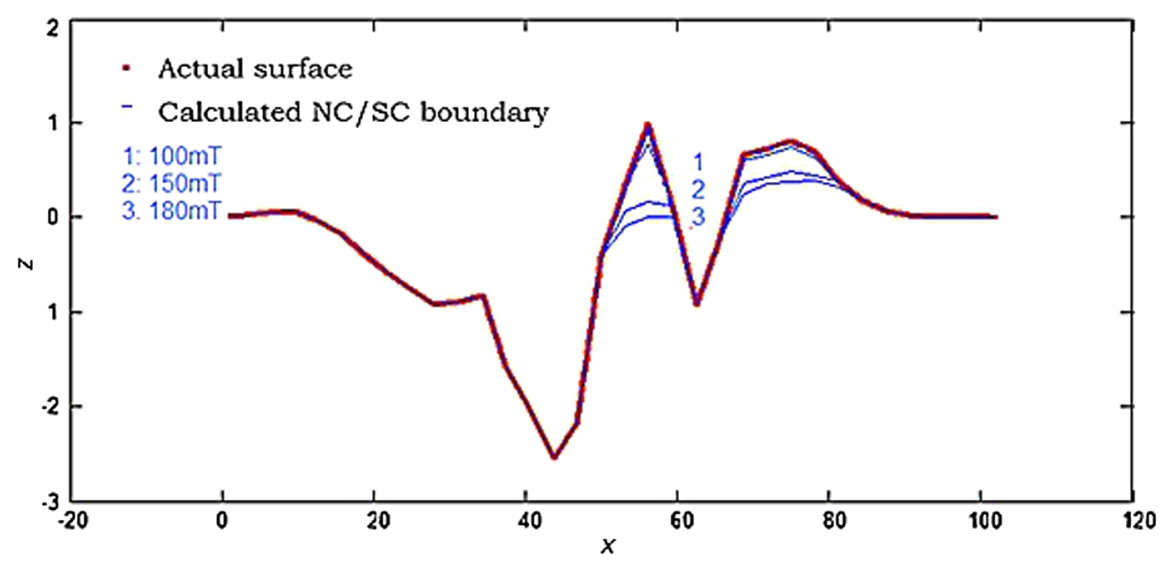

FIG. 7. Normal conducting phase fronts as calculated from different excited fields. Unit: $\mu \mathrm{m}$. 
With the first order solution, the thermal conductivity is a constant because the temperature difference on the surface is small. Equation (7) further reduces into a Poisson equation:

$$
\alpha(T) \nabla^{2} T=-\tilde{q}=-\frac{\tilde{Q}(t, x, y, z)}{\rho C_{P}} .
$$

The right term $\tilde{q}$ in Eq. (8) is treated as a dynamic source, the rf power loss at a given field. The thermal diffusion time constant $t$ is determined by $l^{2} / \alpha$. The $t$ is characteristic size, which is $100 \mu \mathrm{m}$. The $\alpha$ is the thermal diffusivity, which is $5000 \mathrm{~cm}^{-2} \mathrm{sec}^{-1}$ at $4 \mathrm{~K}$ [22]. Therefore, the thermal diffusion time constant is of order $10^{-7}$ second. This means temperature change is slow compared with the rf field changes. The $\tilde{q}$ in Eq. (8) may then be an averaged thermal source, and the temperature map at an equilibrium state is calculated at a given field amplitude.

The thermal simulation setup is illustrated in Fig. 8. The vertical simulated length is $3.3 \mathrm{~mm}$, which is a typical cavity wall thickness. To confidently model the temperature map in a bounded area, the horizontal scale needs to be comparable to the cavity thickness. If the lateral length is set too small, the simulation leads to temperature calculation error because both side boundaries are heat isolation conditions. However, setting the lateral zone too large costs computation inefficiency. We take a lateral length of $6.6 \mathrm{~mm}$ in our simulation to simulate the thermal response of an isolated defect region under typical cavity cooling conditions. The geometry adaptive meshing technique computationally focuses attention on surface roughness

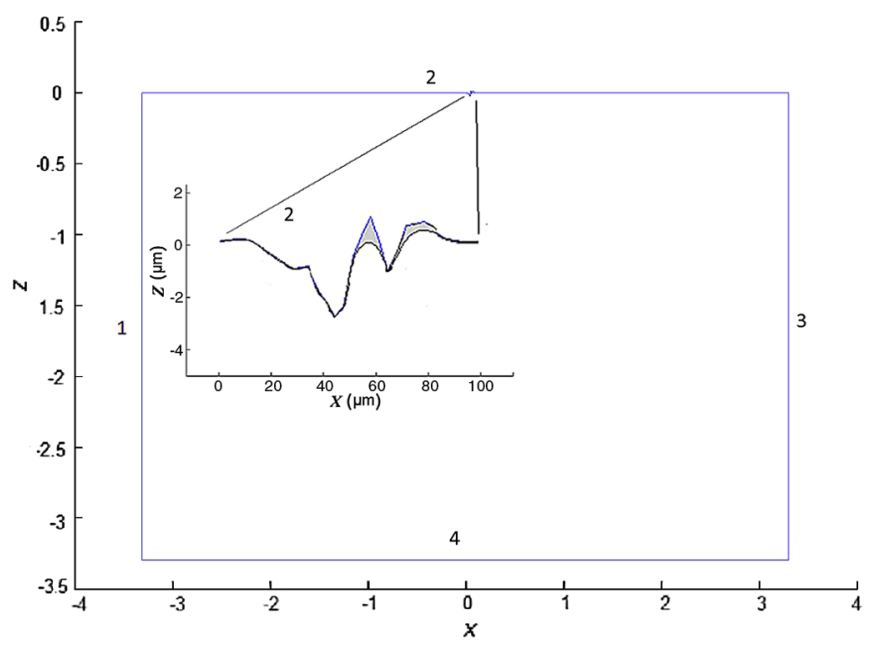

FIG. 8. Typical temperature simulation area in 2D dimension: Borders are labeled in numbers. Borders 2 and 4 are rf surface and helium interfaces respectively, and borders 1 and 3 have thermal isolation boundary conditions. Inset: The surface roughness feature is highlighted. The grey area represents the internal heat source location. Unit: $\mu \mathrm{m}$. features because the area ratio between roughness features (inserted) and the whole simulated area is small [23].

Boundary conditions are illustrated in Fig. 8. Borders 1 and 3 satisfy Neumann boundary conditions. Border 2 is the rf surface. The inset in the figure is an enlargement where the isolated surface feature for assessment with a lateral scale of $100 \mu \mathrm{m}$ is located on the center of border 2 . The grey area shows the heat source zone. The convection cooling boundary condition is applied at border 4. Mathematically, it is a Robin or absorption boundary condition (ABC), and it can be expressed as below [24]:

$$
\kappa \frac{\partial T}{\partial n}=h_{\text {Kap }}\left(T-T_{\text {bath }}\right)=\tilde{q},
$$

where $\kappa$ is thermal conductivity, and $h_{\text {Kap }}$ is the Kapitza conductivity between helium and $\mathrm{Nb}$. Both $h_{\text {Kap }}$ and $\kappa$ are temperature dependent. These dependencies are given in Eq. (10) $[25,26]$ :

$$
\begin{aligned}
\kappa(T)= & 0.7 e^{1.65 T-0.1 T^{2}}\left(\frac{W}{K \cdot m}\right) \\
h_{\text {Kap }}\left(T, T_{0}\right)= & 200 \cdot T^{4.65}\left[\begin{array}{c}
1+1.5\left(\frac{T-T_{0}}{T_{0}}\right)+\cdots \\
\cdots+\left(\frac{T-T_{0}}{T_{0}}\right)^{2}+0.25\left(\frac{T-T_{0}}{T_{0}}\right)^{3}
\end{array}\right] \\
& \times\left(\frac{W}{K \cdot m^{2}}\right) T-T_{0}<1.4 \mathrm{~K} .
\end{aligned}
$$

Next, we further consider the heat source term $\tilde{Q}$ in Eq. (8). Note that the commonly used surface area integration of Eq. (11) is applicable only if one presumes that the $H$ field homogeneously penetrates the uniform surface within a skin depth. Our simulation is an unusual circumstance because the normal conducting dissipative layers are thinner than the normal conducting skin depth, unlike an assumption taken in [5]. This means Eq. (11) is not suitable for the loss calculation here:

$$
\tilde{Q}=\int \frac{1}{2} \times R_{\text {surface }} \times H^{2} d S
$$

Since this assumption is not valid in our simulation case, the rf dissipated from a small normal zone volume should be an integration based on the local electric field and electrical conductivity as in Eq. (12):

$$
\tilde{Q}=\int \frac{1}{2} \times \sigma \times E^{2} d V
$$

where $\sigma$ is electric conductivity, $E$ is the volume electric field, and the integration $\tilde{Q}$ is the loss in the volume of the normal zone.

The electric field in the normal zone may be calculated from a quasistatic increasing $H$ field from MaxwellFaraday law in Eq. (13): 


$$
\begin{aligned}
& \frac{\partial E_{z}}{\partial y}=\omega \mu H_{x} \\
& \frac{\partial E_{z}}{\partial x}=-\omega \mu H_{y} .
\end{aligned}
$$

Note, in our model the electric field lies in a direction perpendicular to the plane of the paper and has integrated amplitude described by Eq. (14):

$$
\begin{aligned}
E_{z}(x, y)= & \omega \mu \sin \omega t\left(\int_{y_{0}}^{y}-\frac{\partial \varphi}{\partial x} d y+\int_{x_{0}}^{x} \frac{\partial \varphi}{\partial y} d x\right) \\
& +E_{0}\left(x_{0}, y_{0}\right),
\end{aligned}
$$

where electric field $E_{0}\left(x_{0}, y_{0}\right)$ is $E$ field on the normal conducting and superconducting interface. Its value is set to zero. Equations (13) and (14) suggest that rf power loss is proportional to $\omega^{2}$.

Numerically, rf power loss is calculated in the form of discrete power density on each element. This loss is the input for the thermal simulation. Compared to the rf loss in the normal zone, the rf loss from the superconducting zone is small and neglected at this stage. Thermal conductivity is updated locally after each iteration until a temperature map converges on each element. The algorithm is illustrated in the flowchart in Fig. 9. With a converged temperature map, the resulting rf loss is expressed by an effective surface resistance.

\section{Simulation results and comparison}

Using the results described in Fig. 7, the calculation results of the consequent temperature map inside the cavity wall are demonstrated in Fig. 10. The simulated setup configuration is from the model of Fig. 8, and the results in Fig. 10 are at two different applied $H$ field levels.

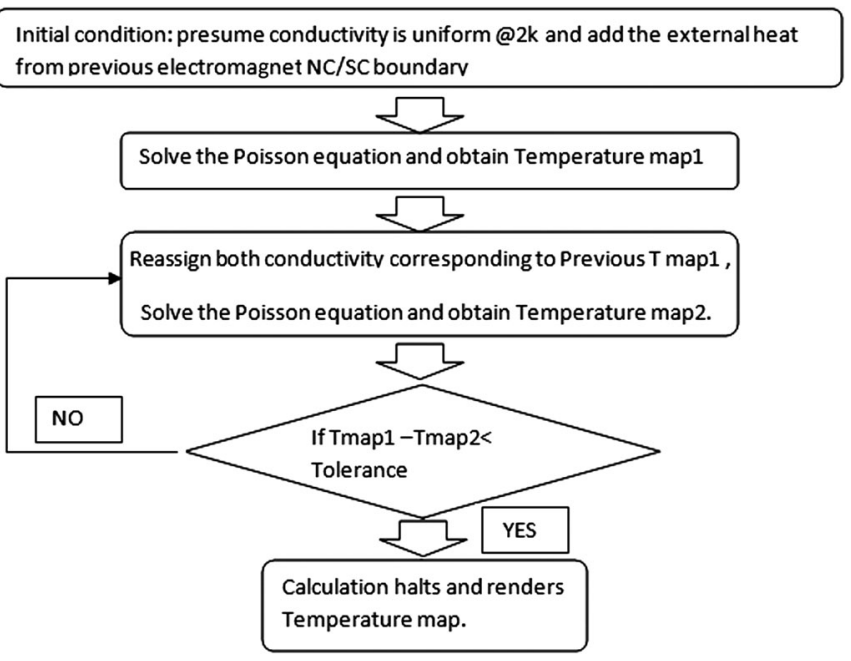

FIG. 9. The flowchart of thermal equation simulation. It is used to calculate the temperature distribution.

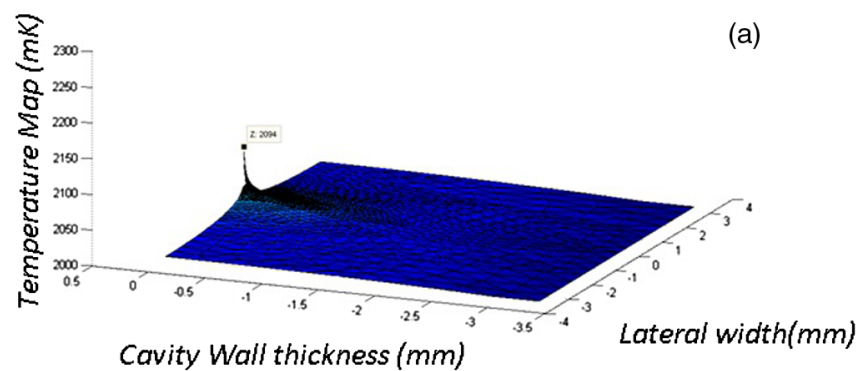

(b)

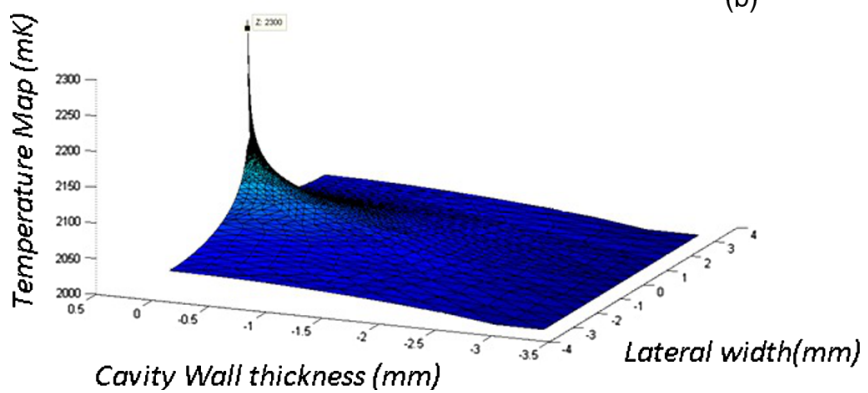

FIG. 10. Temperature maps calculated for applied magnetic field of (a) $100 \mathrm{mT}$ and (b) $120 \mathrm{mT}$ with the isolated $100 \mu \mathrm{m}$ rough strip from Fig. 8. The maximum temperature reaches 2.094 and $2.300 \mathrm{~K}$, respectively. The helium bath condition is $2.00 \mathrm{~K}$.

In Fig. 10(b), the radius of significantly heated zones on the surface can be as large as $\mathrm{mm}$ scale from a localized feature. With an exciting field of $100 \mathrm{mT}$, the highest temperature is calculated to be $94 \mathrm{mK}$ higher than the helium bath temperature. At an exciting field of $120 \mathrm{mT}$, the highest temperature is around $300 \mathrm{mK}$ higher than the helium bath temperature. These temperatures are far below the $\mathrm{Nb}$ transition temperature $9.2 \mathrm{~K}$, suggesting that there is no significant thermal correction on the normal conducting zone size. It is thermally stabilized.

\section{Electromagnetic and thermal iteration simulation}

Superconductivity is bounded by three threshold critical parameters: current, magnetic field, and temperature. Temperature strongly influences the critical transition $H_{c}$ and further defines the normal conducting and superconducting interface location, which in turn determines the effective surface resistance [1]. Fortunately, $H_{c}$ varies little at low temperatures. Hence, this correction has a minor effect on $\mathrm{rf}$ loss estimate. $H_{c}(T)$ is typically corrected below in Eq. (15) [1]:

$$
H_{c}(T)=H_{c}(0 \mathrm{~K})\left[1-\left(\frac{T}{T_{c}}\right)^{2}\right] .
$$

The location of the normal conducting phase front will be corrected numerically by the new temperature. Since the temperature rises at the sharp topographic features, local $H_{c}$ would decrease. Thus, a new electromagnetic and thermal configuration requires a recalculation. Therefore, we need to 


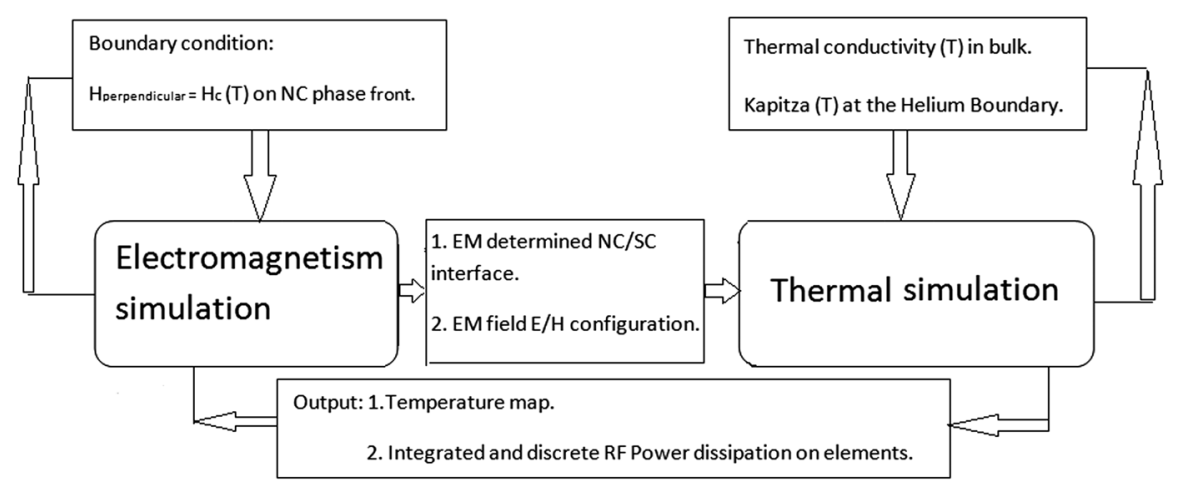

FIG. 11. Flowchart of electromagnetic and temperature simulations. It is used to correct the size of the normal conducting zone and to estimate the rf loss. The flowchart shows a "big" iteration with two "small" iterations. Results of electromagnetic and thermal simulations are detailed in Secs. II A and II B in Figs. 6 and 9.

introduce a thermal feedback model including the $H_{c}(T)$ dependency and generate a higher level iteration that includes both simulations described in Secs. II A and II B [22]. The flowchart of this big iteration is given in Fig. 11.

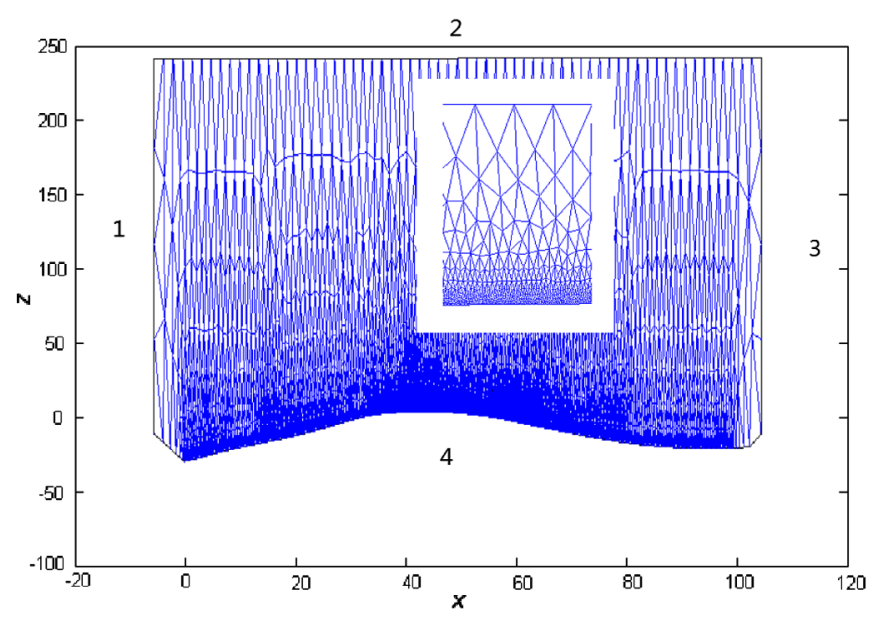

FIG. 12. Electromagnetic calculation model for electropolished $\mathrm{Nb}$ surface. Note: The axis ratio is set as 3:1 to show in a distinguishable format. The inset is an enlargement where the axis ratio is $1: 1$. Unit: $\mu \mathrm{m}$.

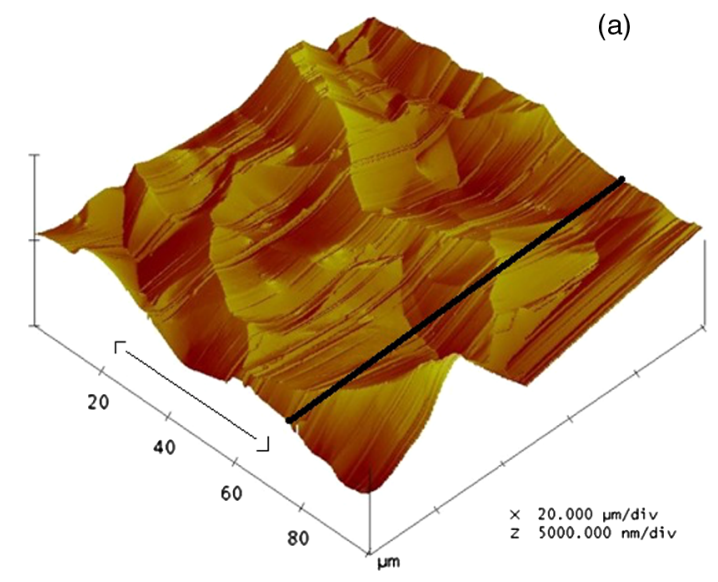

Similar to the representative BCP-treated $\mathrm{Nb}$ surface in Fig. 2, an electropolished (EP) fine grain $\mathrm{Nb}$ surface was characterized by a $100 \mu \mathrm{m}$ AFM scan and is plotted in Fig. 12. The same FEM calculation was conducted with the same boundary conditions described in Fig. 2, only exchanging boundary 4 with the representative EP surface profile. For this simulation, a geometry adaptive meshing was used to accommodate the fine surface features. The inset in the figure is an enlargement of meshing elements on the center of boundary 4 with an equal axis ratio.

\section{APPLICATION TO CHARACTERISTIC ETCHED AND ELECTROPOLISHED NB SURFACE TOPOGRAPHIES}

The described integrated analysis above was applied to two 3D AFM profiles from BCP- and EP-treated fine grain $\mathrm{Nb}$ surfaces. Such representative surfaces can be replicated from cavities without undermining their performances [27-29]. The AFM scans used in this analysis are plotted in Fig. 13. The AFM characterization area covers $100 \times 100 \mu \mathrm{m}$. The effective raster strip width depends on the sampling rate, which is $512 \times 512$ in our case.

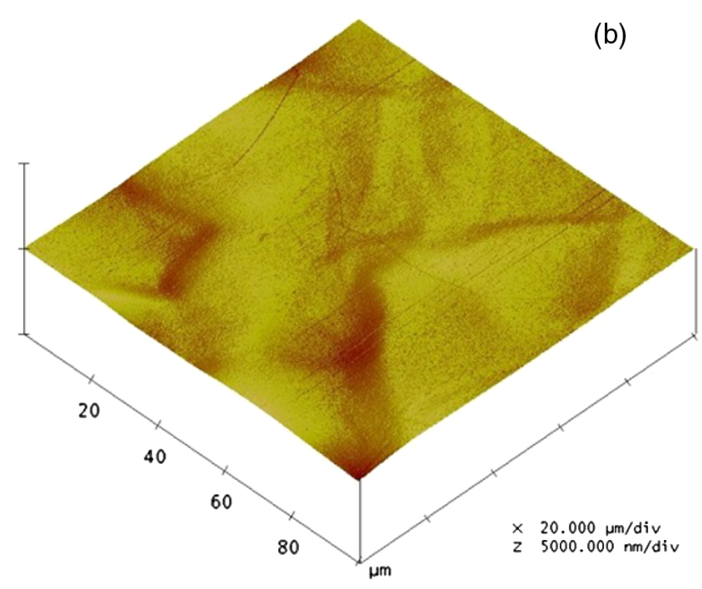

FIG. 13. AFM images from a fine grain niobium sample with (a) $\sim 100 \mu \mathrm{m}$ removal by BCP, (b) after electropolished at $30{ }^{\circ} \mathrm{C}$ to remove $48 \mu \mathrm{m}$. Horizontal scale is $20 \mu \mathrm{m}$ per division and vertical scale is $5 \mu \mathrm{m}$ per division [3]. 


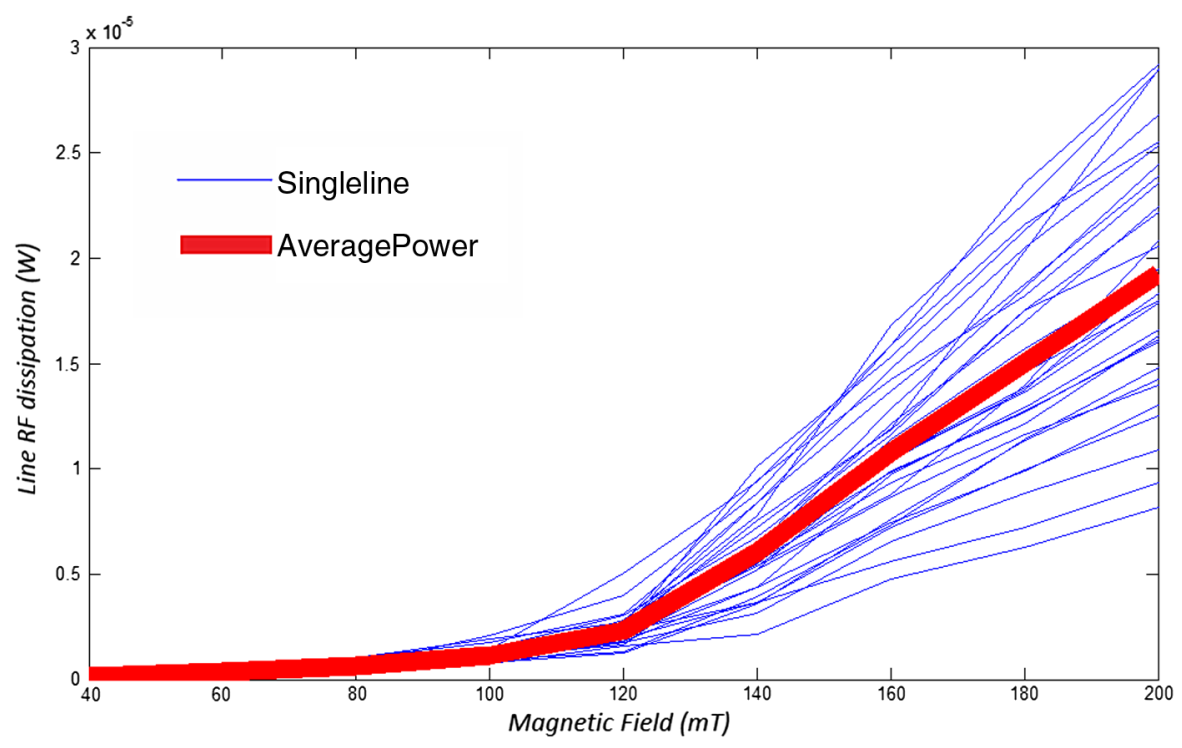

FIG. 14. Calculated rf power dissipation on each representative $3.125 \mu \mathrm{m}$ wide strip as a function of external applied magnetic $H$ field for a $100 \mu \mathrm{m} \times 100 \mu \mathrm{m}$ BCP-treated fine grain $\mathrm{Nb}$ surface. Blue lines are the rf loss from each of 32 strips and the red line is the averaged rf loss.

Limited by computational capacity, we reduce the resolution to $32 \times 32$. As a result, strip columns, represented in Figs. 2 and 12, are taken to represent a width of $3.125 \mu \mathrm{m}$. The black line in Fig. 13(a) marks such a typical strip. In this analysis, rf losses are then collected from the normal zones along $100 \mu \mathrm{m} \times 3.125 \mu \mathrm{m}$ strips, and the resulting effective surface resistance increase from topographical field enhancements is calculated.

The rf losses on 32 such columns derived from the BCP surface profile were thus calculated as a function of applied $H$ fields. Figure 14 indicates the rf loss increasing with field due to the expansion of the small normal conducting zones. The losses from the individual strips (shown in blue) are calculated from Eq. (12). The averaged rf loss from these 32 strips is taken as representative of that due to the typical surface topography of a fine grain $\mathrm{Nb}$ surface that has been etched by BCP. Note that all losses in the superconducting zones have been neglected here.

Figure 15 shows the average loss from an EP surface derived from the same analysis method as that shown in Fig. 14 for the BCP'd surface. Note the dramatic difference in calculated field-dependent losses from $\mathrm{Nb}$ surfaces etched by BCP and EP. These losses are collected from microscopic thermally stabilized normal conducting regions. Comparison of these two surfaces suggests that a significant density of small normal conducting zones is

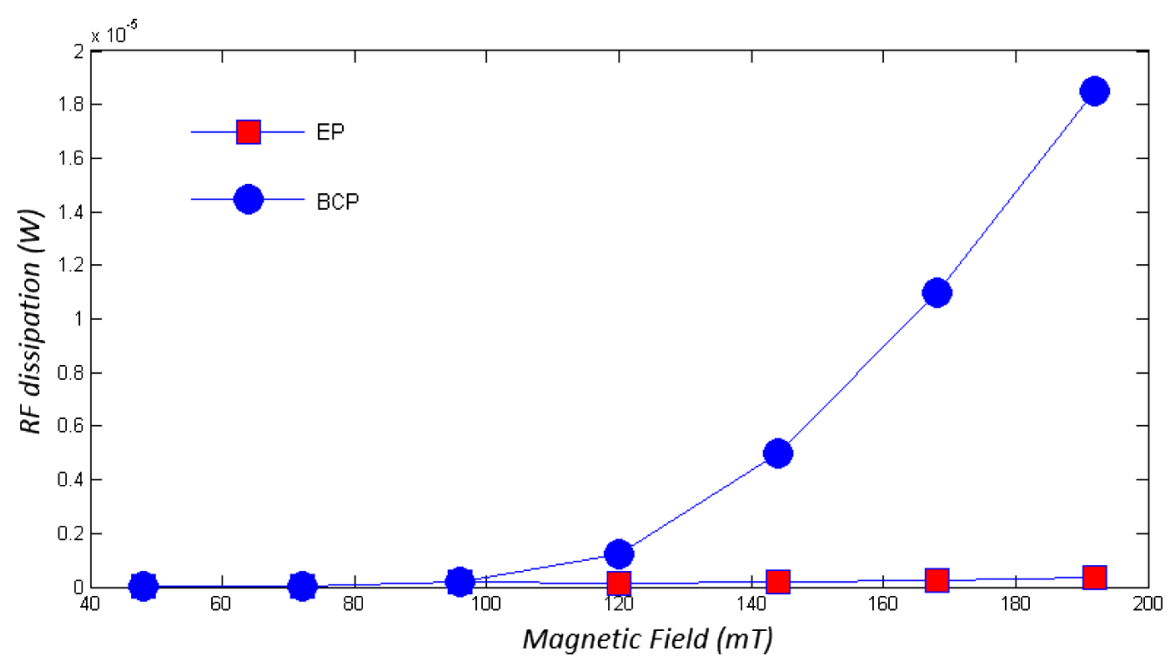

FIG. 15. Average topography-induced power dissipation on $100 \mu \mathrm{m} \times 100 \mu \mathrm{m}$ BCP and EP surfaces plotted as a function of peak applied $H$ field. Superconducting state losses are ignored. 


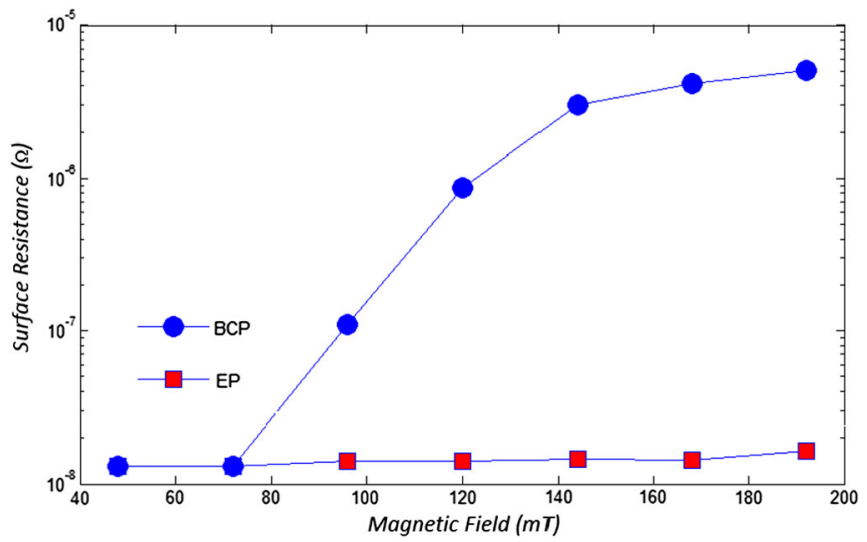

FIG. 16. Simulated effective rf surface resistance with peak $H$ field of $\mathrm{BCP}$ - and $\mathrm{EP}$-treated fine grain $\mathrm{Nb}$ surfaces, including representative constant-temperature superconducting state losses.

generated on $\mathrm{BCP}$ surfaces, while few normal zones are generated on EP-treated surfaces. The additional heat generated on the EP-treated surface in this simulation is not significantly different from an ideally flat surface.

If the sum of rf losses on the 32 strips is represented by $\tilde{Q}$, the effective surface resistance is then

$$
R_{\text {surface }}=\frac{2 \tilde{Q}}{H^{2} \int d s} .
$$

This effective surface resistance is therefore proportionally related to the loss and density of microscopic fieldinduced normal zones. Now adding a small superconducting state resistance of $\sim 13 \mathrm{n} \Omega$, we obtain the resulting effective surface resistance as illustrated in Fig. 16.

\section{DISCUSSION}

We now consider how such nonlinear surface resistance would be reflected in the performance of a typical SRF accelerating cavity. Allowing that the local effective surface resistance has field dependency as described in Fig. 16, we integrate the $\mathrm{rf}$ loss of a resonator cavity by Eq. (17):

$$
P=\frac{1}{2} \times \int_{\substack{\text { Cauity } \\ \text { Surface }}} R(|H|) \times H(r, z)^{2} d S(r, z) .
$$

In a representative elliptical $\beta=1$ accelerating cavity, taking the approximation that the amplitude of surface $H$ field is zero in the regions near irises and maximum along the equators, we can derive the effective surface resistance from the integration in Eq. (17). Then, the quality factor can be calculated from the simple expression of Eq. (18):

$$
\begin{aligned}
Q_{\substack{\text { topo } \\
\text { heat }}}(H) & =\frac{\omega_{0} U}{P}=\frac{\frac{1}{2} \omega_{0} \mu_{0} \int_{v} H^{2} d v}{\frac{1}{2} R_{\substack{\text { Surface } \\
\text { Resistance }}}(|H|) \times \int_{s} H^{2} d s} \\
& \cong \frac{G}{R_{\substack{\text { Surface } \\
\text { Resistance }}}(H)} .
\end{aligned}
$$

The "low loss" cell geometry used in the CEBAF $12 \mathrm{GeV}$ upgrade seven-cell $\mathrm{C} 100$ cavity has been simulated in SUPERFISH [30]. The normalized surface $H$ field amplitude profile obtained is illustrated in Fig. 17. In Fig. 17, the blue curve is the cavity profile, the red curve is the surface $H$ field, and the dashed curve is our simplified surface field.

The geometry factor for this structure in this accelerating mode is $\sim 280 \Omega$ and $B_{\text {peak }} / E_{\text {acc }}$ value is $3.74 \mathrm{mT} /$ $(\mathrm{MV} / \mathrm{m})$. The quality factor of such a cavity with a correction for the interior surface topographic effect is given in Eq. (19),

$$
Q_{\text {calc }}=\frac{G}{R_{\text {surface }}(H)}=\frac{G}{R_{B C S}+R_{\text {topo }}(H)}
$$

where the surface resistance comprises $R_{\text {topo }}(H)$ from Fig. 16 and BCS resistance. The BCS surface resistance is presumed to have no field dependency for first order simplicity. At $1.5 \mathrm{GHz}, R_{\mathrm{BCS}}$ is commonly $\sim 13 \mathrm{n} \Omega$ at $2 \mathrm{~K}$, while the topographically induced surface resistance is zero below some threshold field level. Consequently, the quality factor, $Q$, is dominated by BCS resistance at low fields. Figure 18 shows the quality factors, $Q_{0}$, as a function of surface $H$ field predicted by this analysis that would correspond to a low loss shaped cavity having $\mathrm{Nb}$ surface topography represented by the sampled two different surface treatments, BCP and EP. Note that thermal feedback on the surface resistance of the superconducting material has not been included; this would, of course, result in even further nonlinear reduction of $Q_{0}$.

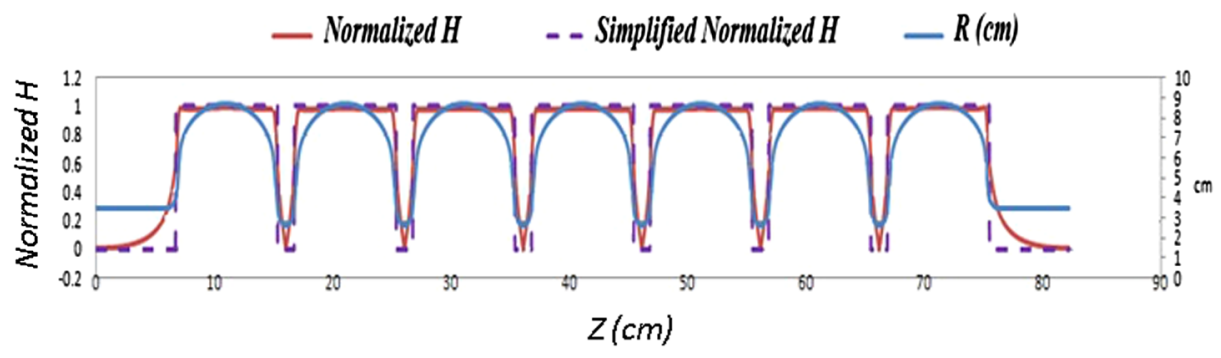

FIG. 17. Surface magnetic field on CEBAF C100 seven-cell cavity from SuPERFISH. 


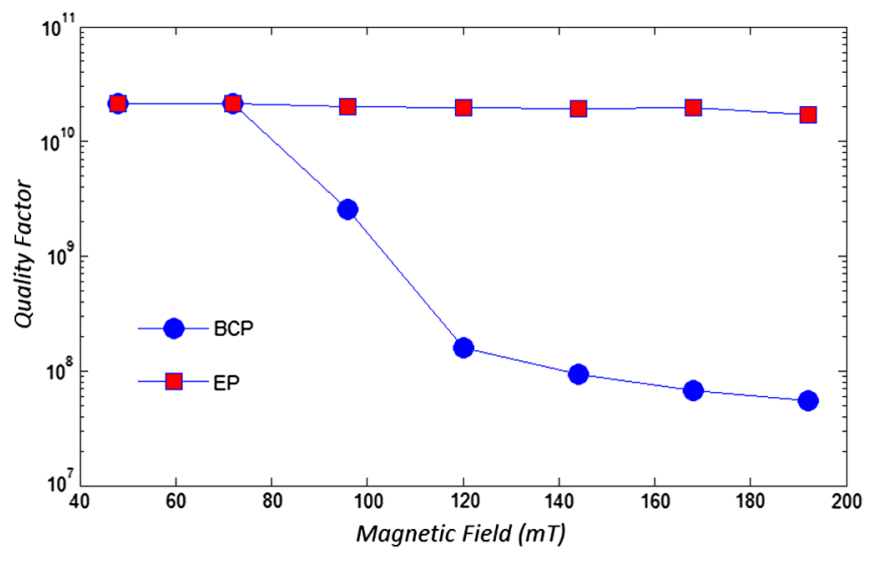

FIG. 18. Comparison of the calculated effective cavity quality factor simulated for representative fine grain $\mathrm{Nb}$ BCP'd and EP'd surfaces at different peak $H$ fields in a C100 geometry cavity. Thermal feedback effects are not included.

As the normal conducting zones grow, some of the simplifying assumptions in our present analysis break down, the superconducting material losses become nonnegligible, and the $Q$ decreases even faster than has been modeled here.

The model calculation results for the representative BCP-etched surface are in Fig. 19 plotted together with $2 \mathrm{~K}$ performance test data for a Jefferson Lab upgrade prototype seven-cell cavity having this low loss geometry (LL002), both heavily BCP etched and subsequently electropolished [31,32]. Although one will certainly seek higher resolution from future model calculations, there is rough quantitative agreement between the calculation predictions and observed cavity performance in this case

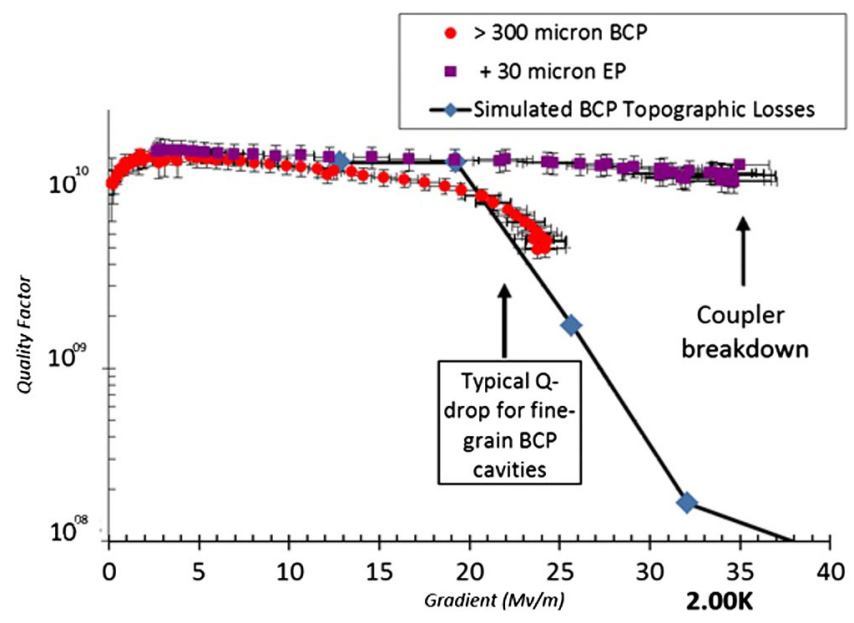

FIG. 19. Comparison of model-predicted $Q_{0}$ having representative BCP surface topography with experimental data for CEBAF prototype cavity LL002 having heavily BCP etched and subsequent electropolished surfaces. No evidence of field emission loading was observed in either test. of a heavily etched cavity. This is consistent with the interpretation that topographical field enhancements are the cause of these enhanced nonlinear losses on BCP-etched fine grain niobium, i.e. $Q$-drop from fine-scale roughness. Such field enhancements are absent from appropriately electropolished surfaces, so that this $Q$-drop mechanism is absent for EP-treated cavities.

Since the specific details of the surface structure of etched $\mathrm{Nb}$ surfaces (in contrast to electropolished surfaces) depend strongly on residual strains and defect densities, as well as the amount of etching removal from an otherwise smooth surface $[33,34]$, one should not be surprised to encounter significant variation of the topography-induced rf losses in different circumstances, though the phenomenon should be universal. One may, for example, interpret the small but significant systematic $Q$-drop reported at the high-field limit of the subset of XFEL cavities which received a final light $\mathrm{BCP}$ etch [35] as attributable to the low-amplitude sharpening of crystallographic edges creating widely dispersed local field enhancements.

\section{SUMMARY}

Extending the analysis begun by [5], simplified electromagnetic and thermal simulations have been developed to analyze the microscopic scale geometric surface field enhancement and the normal/superconducting material interface when the local field exceeds $H_{c}$. The location of this interface phase front is a function of the exciting magnetic field and the specific topography. The thermally stable normal zone areas contribute significantly to the rf power loss. Each normal zone initiates its expansion based on the local geometric field enhancement factor. More accurate normal zone phase front modeling is obtained considering the critical field's temperature dependence. The volume of the normal zone on the surface expands nonlinearly with increasing external magnetic field. Such nonlinearity and the corresponding increase in dissipative rf power can be represented by an effective nonlinear surface resistance. Additional superconducting rf loss is also induced as a result of thermal feedback. The total $\mathrm{rf}$ power loss contribution thus induced solely by topographic roughness may be calculated. Initial results of this analysis using representative topographic profile data from typical $\mathrm{BCP}$ etched and EP fine grain $\mathrm{Nb}$ surfaces yield a nonlinear loss character, and the $Q$ dependence with field is quite similar to that typically observed with L-band SRF accelerating cavities with the corresponding surface treatments. This suggests that an adequate explanation for the fielddependent differences in BCP'd and EP'd fine grain $\mathrm{Nb}$ cavities is now in hand. Opportunities to improve the precision of this model calculation have been identified, but physical insight into the phenomenon linking microscopic surface topography to high-field loss character of niobium SRF cavities for accelerators has been significantly enhanced. 


\section{ACKNOWLEDGMENTS}

This material is based on work supported by the U.S. Department of Energy, Office of Science, Office of Nuclear Physics under Contract No. DE-AC05-06OR23177. C. X. is grateful for discussions with Alexander Gurevich in Old Dominion University, Haipeng Wang, Hui Tian and Olga Trofimova at Jefferson Lab, John Delos at College of William and Mary and Toby Driscoll at University of Delaware.

[1] H. Padamsee, J. Knobloch, and T. Hays, RF Superconductivity for Accelerators, 2nd ed. (Wiley and Sons, New York, 2008).

[2] C.E. Reece and G Ciovati, Superconducting radiofrequency technology R\&D for future accelerator applications, Rev. Accel. Sci. Techol. 05, 285 (2012).

[3] C. Xu, H. Tian, C. E. Reece, and M. J. Kelley, Enhanced characterization of niobium surface topography, Phys. Rev. ST Accel. Beams 14, 123501 (2011).

[4] C. Xu, H. Tian, C. E. Reece, and M. J. Kelley, Topographic power spectral density study of the effect of surface treatment processes on niobium for superconducting radio frequency accelerator cavities, Phys. Rev. ST Accel. Beams 15, 043502 (2012).

[5] J. Knobloch, R. L. Geng, M. Liepe, and H. Padamsee, High-field Q slope in superconducting cavities due to magnetic field enhancement at grain boundaries, in Proceedings of the 9th Workshop on RF Superconductivity (Los Alamos National Laboratory, Santa Fe, NM, 1999), pp. 77-91.

[6] T. Kubo, Magnetic field enhancement at a pit on the surface of a superconducting accelerating cavity, Prog. Theor. Exp. Phys. 2015, 073 G01 (2015).

[7] E. Kako, S. Noguchi, M. Ono, K. Saito, T. Shishido, H. Safa, J. Knobloch, and L. Lilje, Improvement of cavity performance in the Saclay/Cornell/DESY's SC cavities, in Proceedings of the 9th Workshop on RF Superconductivity (Los Alamos National Laboratory, Santa Fe, NM, 1999), pp. 179-186.

[8] K. Saito, S. Noguchi, H. Inoue, M. Ono, T. Shishido, Y. Yamazaki, N. Ouchi, J. Kusano, M. Mizumoto, and M. Matsuoka, Superiority of electropolishing over chemical polishing on high gradients, in Proceedings of the 8th International Workshop on RF Superconductivity (JACoW, Abano Terme, Italy, 1997), pp. 534-539.

[9] K. Saito, Development of electropolishing technology for superconducting cavities, in Proceedings of the 2003 Particle Accelerator Conference, Portland, OR (IEEE, New York, 2003), pp. 462-466.

[10] D. Reschke, S. Aderhold, A. Gössel, J. Iversen, S. Karstensen, D. Kostin, G. Kreps, A. Matheisen, W.-D. Möller, F. Schlander, W. Singer, X. Singer, N. Steinhau Kühl, A. A. Sulimov, and K. Twarowski, Results on large grain nine-cell cavities at DESY: gradients up to $45 \mathrm{MV} / \mathrm{m}$ after electropolishing, in Proceedings of the 15th International Conference on $R F$ Superconductivity (JACoW, Chicago, IL, 2011), pp. 490-494.
[11] H. Tian and C.E. Reece, Quantitative EP studies and results for SRF Nb cavity production, in Proceedings of the 15th International Conference on RF Superconductivity (JACoW, Chicago, IL, 2011), pp. 565-570.

[12] C. E. Reece, A. C. Crawford, and R. L. Geng, Improved performance of JLab 7 cell cavities by electropolishing, in Proceedings of the 23rd Particle Accelerator Conference, Vancouver, Canada, 2009 (IEEE, Piscataway, NJ, 2009), pp. 2126-2128.

[13] T. F. Stromberg and C. A. Swenson, Negative Surface FreeEnergy Effects in Superconducting Niobium, Phys. Rev. Lett. 9, 370 (1962).

[14] S. Posen, N. Valles, and M. Liepe, Radio Frequency Magnetic Field Limits of $\mathrm{Nb}$ and $\mathrm{Nb}_{3} \mathrm{Sn}$, Phys. Rev. Lett. 115, 047001 (2015).

[15] T. Kubo, Field limit and nano-scale surface topography of superconducting radio-frequency cavity made of extreme type II superconductor, Prog. Theor. Exp. Phys. 2015, 063G01 (2015).

[16] S. Berry, C. Z. Antoine, and M. Desmons, Surface morphology at the quench site, in Proceedings of the 9th European Particle Accelerator Conference, Lucerne, 2004 (EPS-AG, Lucerne, 2004), pp. 1000-1002.

[17] V. Shemelin and H. Padamsee, Tesla Technology Collaboration Report No. 2008-07, Hamburg, Germany, 2008.

[18] A. Dzyuba, A. Romanenko, and L. D. Cooley, Model for initiation of quality factor degradation at high accelerating fields in superconducting radio-frequency cavities, Supercond. Sci. Technol. 23, 125011 (2010).

[19] S. Kim and I. E. Campisi, Thermal stabilities and optimal operating parameters for the Oak Ridge Spallation Neutron Source superconducting linear accelerator, Phys. Rev. ST Accel. Beams 10, 032001 (2007).

[20] J. D. Jackson, Classical Electrodynamics, 3rd ed. (Wiley, New York, 1998).

[21] S. I. Barry and J. Caunce, Exact and numerical solutions to a Stefan problem with two moving boundaries, Appl. Math. Model. 32, 83 (2008).

[22] J. E. Jensen, W. A. Tuttle, R. B. Stewart, H. Brechna, and A. G. Prodell, Brookhaven National Laboratory Report No. 10200, Volume II, 1980.

[23] T. Plewa, T. Linde, and V. Gregory Weirs, Adaptive Mesh Refinement, Theory and Application (Springer, Chicago, 2003).

[24] K. Warnick, Numerical Methods for Engineering: An Introduction Using MATLAB and Computational Electromagnetics (SciTech Publishing, Provo, UT, 2011).

[25] P. Bauer, N. Solyak, G. L. Ciovati, G. Eremeev, A. Gurevich, L. Lilje, and B. Visentin, Evidence for non-linear BCS resistance in SRF cavities, Physica (Amsterdam) 441C, 51 (2006).

[26] K. Mittag, Kapitza conductance and thermal conductivity of copper niobium and aluminium in the range from 1.3 to $2.1 \mathrm{~K}$, Cryogenics 13, 94 (1973).

[27] C. Xu, C. Reece, and M. Kelley, Characterization of Nb SRF cavity materials by white light interferometry and replica techniques, Appl. Surf. Sci. 274, 15 (2013).

[28] S. Berry, C.Z. Antoine, A. Aspart, J. P. Charrier, M. Desmons, and L. Margueritte, Topologic analysis of samples and cavities: A new tool for morphologic inspection of quench site, in Proceedings of the 11th Workshop on RF 
Superconductivity (JACoW, Lubeck, Germany, 2003), pp. 591-593.

[29] M. Ge, G. Wu, D. Burk, J. Ozelis, E. Harms, D. Sergatskov, D. Hicks, and L. D. Cooley, Routine characterization of 3D profiles of SRF cavity defects using replica techniques, Supercond. Sci. Technol. 24, 035002 (2011).

[30] J. Sekutowicz, G. Ciovati, P. Kneisel, G. Wu, A. Brinkmann, R. Parodi, W. Hartung, and S. Zheng, Cavities for JLab's $12 \mathrm{GeV}$ upgrade, in Proceedings of the 2003 Particle Accelerator Conference, Portland, OR, pp. 1395-1397.

[31] C. E. Reece, E. F. Daly, S. Manning, R. Manus, S. Morgan, J. P. Ozelis, and L. Turlington, Fabrication and testing of the SRF cavities for the CEBAF $12 \mathrm{GeV}$ upgrade prototype cryomodule Renascence, in Proceedings of the $21 \mathrm{st}$ Particle Accelerator Conference, Knoxville, TN, 2005 (IEEE, Piscataway, NJ, 2005), pp. 4081-4083.

[32] C. E. Reece and H. Tian, Exploiting new electrochemical understanding of niobium electropolishing for improved performance of SRF cavities for CEBAF, in Proceedings of the 25th International Linear Accelerator Conference, LINAC-2010, Tsukuba, Japan (KEK, Tsukuba, Japan, 2010), pp. 779-781.

[33] X. Zhao, G. Ciovati, C. E. Reece, and A. T. Wu, Surface topography of "hotspot" regions from a single cell SRF cavity, in Proceedings of the 23rd Particle Accelerator Conference, Vancouver, Canada, 2009, pp. 2147-2149.

[34] X. Zhao, G. Ciovati, C. E. Reece, and A. T. Wu, Study of etching pits in a large-grain single cell bulk niobium cavity, in Proceedings of the 2009 International Conference on RF Superconductivity (JACoW, Berlin, Germany, 2009), pp. 446-448.

[35] N. Walker, D. Reschke, J. Schaffran, L. Steder, M. Wiencek, and I. Monaco, Update and status of vertical test results of the European XFEL series cavites, in Proceedings of the 2015 International Conference on RF Superconductivity (Whistler, BC, Canada, 2015). 